J. Phys. IV France 127 (2005) 205-210

(C) EDP Sciences, Les Ulis

DOI: $10.1051 / \mathrm{jp} 4: 2005127032$

\title{
Laser femtoseconde, filamentation, nuage et orage
}

\author{
F. Courvoisier, V. Boutou, J. Kasparian, E. Salmon, G. Méjean, \\ J. Yu et J.-P. Wolf
}

Laboratoire de Spectrométrie lonique et Moléculaire (LASIM), UMR CNRS 5579, Université

Claude Bernard Lyon 1, 43 Bd. du 11 Novembre, 69622 Villeurbanne Cedex, France

\begin{abstract}
Résumé. Les applications telles que le contrôle de foudre grâce aux filaments autoguidés générés par un laser femtoseconde nécessitent de propager un tel filament à travers des aérosols de gouttes d'eau. Nous montrons qu'un filament survit à son interaction avec une goutte de diamètre comparable au sien $(95 \mu \mathrm{m})$, ainsi qu'à des nuages d'épaisseur optique 3,2, soit 5\% de transmission. Cette transmission est permise par la présence d'un "bain de photons" autour du filament. Ce bain forme un réservoir contenant une part importante de l'énergie du faisceau, en équilibre avec le filament, et favorisant son alimentation.
\end{abstract}

\section{INTRODUCTION}

L'idée de contrôler ou d'assister le déclenchement de la foudre à l'aide de lasers est presque aussi ancienne que le laser lui-même [1]. Cette technique permettrait de protéger des sites sensibles tels que des installations électriques ou des aéroports des dégâts directs et indirects causés par la foudre, en particulier les perturbations électromagnétiques. Elle permettrait également de fournir sur demande des éclairs pour des études de géophysique ou des applications de test. Dès les années 70 et 80 , des essais utilisant des lasers nanosecondes ont montré d'importantes limitations, faute de produire de longs canaux ionisés [2]. Plus récemment, de nouvelles perspectives ont été ouvertes avec l'avènement de lasers femtosecondes de forte puissance, qui produisent dans l'atmosphère des filaments ionisés [3,4]. Au côté d'une approche basée sur un laser ultrabref fortement focalisé pour produire un plasma plus dense [5-7] mais d'extension spatiale limitée, l'utilisation de longs filaments autoguidés a été proposée pour court-circuiter les électrodes afin de déclencher et de guider des décharges de haute tension $[8,9]$. Il a ainsi été démontré sur des distances entre électrodes allant jusqu'à $4,5 \mathrm{~m}$ que la tension nécessaire pour produire des décharges est abaissée de $30 \%$ lorsque des filaments sont produits entre les électrodes simultanément avec l'impulsion de haute tension. [9] Les décharges sont alors guidées et se produisent en ligne droite le long des filaments au lieu de suivre le chemin erratique caractéristique des décharges naturelles.

Il n'est pourtant pas immédiat de passer des résultats obtenus en laboratoire à l'échelle du mètre au contrôle de foudre à l'échelle de plusieurs centaines de mètres. Certes, les filaments peuvent se propager sur de longues distances [10], et leur formation a été détectée à une distance de $2 \mathrm{~km}$ [11]. Cependant, hormis les limitations issues de la durée de vie du plasma (quelques microsecondes pour les ions $\mathrm{O}_{2}^{-}[12]$ ), une question pertinente concerne la possibilité de produire de longs filaments continûment ionisés, non pas dans une atmosphère claire, mais sous l'orage : les nuages comme la pluie constituent alors un obstacle majeur à la propagation du laser.

La transmission d'un faisceau laser à travers des nuages ou le brouillard est également essentielle pour des domaines aussi variés que la communication point à point par laser, la détection des polluants atmosphériques par Lidar [13,14], la télémétrie, l'imagerie active, voire pour mettre en évidence des effets de relativité générale dans le champ gravitationnel de la terre [15]. Un moyen prometteur pour remédier aux limites de la transmission optique à travers l'atmosphère, telles que la turbulence, la dispersion ou la diffusion, pourrait être l'utilisation de la filamentation. Ce phénomène a d'abord été observé dans les années 1960 avec des lasers nanoseconde dans des milieux condensés [16,17]. Plus récemment, les lasers 
ultrabrefs et de forte puissance ont permis d'observer un phénomène similaire dans l'air, malgré son faible indice de réfraction non-linéaire [18]. Au-delà d'une puissance dite puissance critique $\left(P_{c} \sim 3 \mathrm{GW}\right.$ dans l'air à $800 \mathrm{~nm}$ ), l'autofocalisation du faisceau par effet Kerr domine la diffraction. Les filaments sont alors générés par un équilibre dynamique entre l'autofocalisation et la défocalisation due au plasma issu de l'ionisation multiphotonique locale de l'air dans le filament. Ces filaments se propagent alors comme des quasi-solitons [19] formant des structures localisées dans le faisceau, avec un diamètre typique de $100 \mu \mathrm{m}$. La forte intensité $\left(\sim 10^{14} \mathrm{~W} / \mathrm{cm}^{2}\right)$ [20] et la densité d'électrons $\left(\sim 10^{15} \mathrm{~cm}^{-3}\right)$ dans le filament modifient le milieu traversé, ce qui pourrait conduire à une moindre sensibilité aux fluctuations locales du milieu. Ainsi, la contribution non-linéaire à l'indice de réfraction induite par l'effet Kerr $\left(\Delta \mathrm{n} \sim 10^{-5}\right)$ est plus grande que celle produite par les fluctuations thermiques.

Les fluctuations thermiques ne sont pourtant pas le seul processus susceptible de perturber la propagation d'impulsions laser ultrabrèves dans l'atmosphère. Les particules d'aérosol, qu'il s'agisse de gouttelettes d'eau ou de poussières, peuvent atteindre une taille de plusieurs dizaines de microns, comparable au diamètre des filaments autoguidés. Nous nous sommes donc intéressés à l'interaction de ces filaments avec des gouttes d'eau [21].

\section{INTERACTION D’UN FILAMENT AVEC UNE GOUTTE ISOLÉE}

Dans une première série d'expériences, nous avons étudié l'interaction d'un filament avec une goutte d'eau isolée. Pour cela, nous avons légèrement focalisé des impulsions laser ultrabrèves $(7 \mathrm{~mJ}$ par impulsion, une durée d'impulsion de $120 \mathrm{fs}$ à $810 \mathrm{~nm}$ ) à l'aide d'un miroir sphérique de $5 \mathrm{~m}$ de distance focale. Nous avons ainsi produit un filament d'environ $150 \mu \mathrm{m}$ de diamètre, se propageant sur une distance de plus de 3 mètres, soit 30 fois la longueur de Rayleigh. Pour une puissance laser donnée, le point de démarrage de la filamentation est stable à quelques centimètres près. Cette stabilité nous a permis de définir ce point comme origine pour définir la distance de propagation $d$.

A $d=1 \mathrm{~m}$, le filament interagit avec une goutte d'eau micrométrique calibrée. Cette goutte, de diamètre $a$, est générée par une buse piézoélectrique synchronisée avec le laser à un taux de répétition de $20 \mathrm{~Hz}$, de manière à produire une goutte nouvelle à chaque impulsion laser. Le diamètre des gouttes est réglable de 30 à $100 \mu \mathrm{m}$, pour reproduire une distribution de taille typique des nuages, en variant la tension et la durée du créneau appliqué sur le cristal piézoélectrique. On définit le paramètre d'impact $b$ comme la distance entre l' axe du filament et le centre de la goutte. L'observation directe au microscope et la diffusion élastique aux petits angles nous ont permis de vérifier que $a$ et $b$ sont très bien reproductibles : $\Delta b / a<0,1$, $\Delta a / a<0,05$. Ce dispositif est le même que celui utilisé précédemment pour observer, avec des impulsions laser de moindre énergie, la génération de plasma nanométrique dans des microgouttes d'eau [22].

Nous avons tout d'abord mesuré le profil d'intensité du faisceau laser en propagation libre, en l'absence de goutte. Comme le montrent les inserts de la Figure 1, seule une fraction de l'énergie du faisceau se trouve dans le filament, tandis que le reste de l'énergie l'entoure et se propage parallèlement à lui, formant un "bain de photons" d'environ $2 \mathrm{~mm}$ de diamètre. Nous avons mesuré l'énergie contenue dans le filament à une distance de propagation $d$ en plaçant un film d'aluminium ou de cellulose dans le faisceau. Ce film bloque le "bain de photons", mais est perforé localement par la haute intensité du filament. L'énergie transmise par la perforation est mesurée par un bolomètre. L'erreur de mesure sur l'énergie causée par les fluctuations dans l'épaisseur du film a été estimée à $\pm 4 \%$ d'un film à l'autre. Mais cette erreur est limitée à $1 \%$ pour une position donnée sur le film.

Au point d'interaction avec la goutte $(d=1 \mathrm{~m})$, le filament contient environ $35 \%$ de l'énergie totale. $1 \mathrm{~m}$ plus loin, cette proportion tombe à $13 \%$. Cette énergie est rendue au "bain de photons", qui se comporte comme un "réservoir d'énergie" [23] en équilibre dynamique avec le filament. En valeur absolue, l'énergie contenue dans le filament (Figure 1) décroît de 2,7 mJ à $d=1 \mathrm{~m}$, à $0,25 \mathrm{~mJ}$ à $d=3 \mathrm{~m}$. Au-delà, l'intensité locale dans le filament ne suffit plus pour perforer le film. Ce point nous permet de définir la fin du filament. 


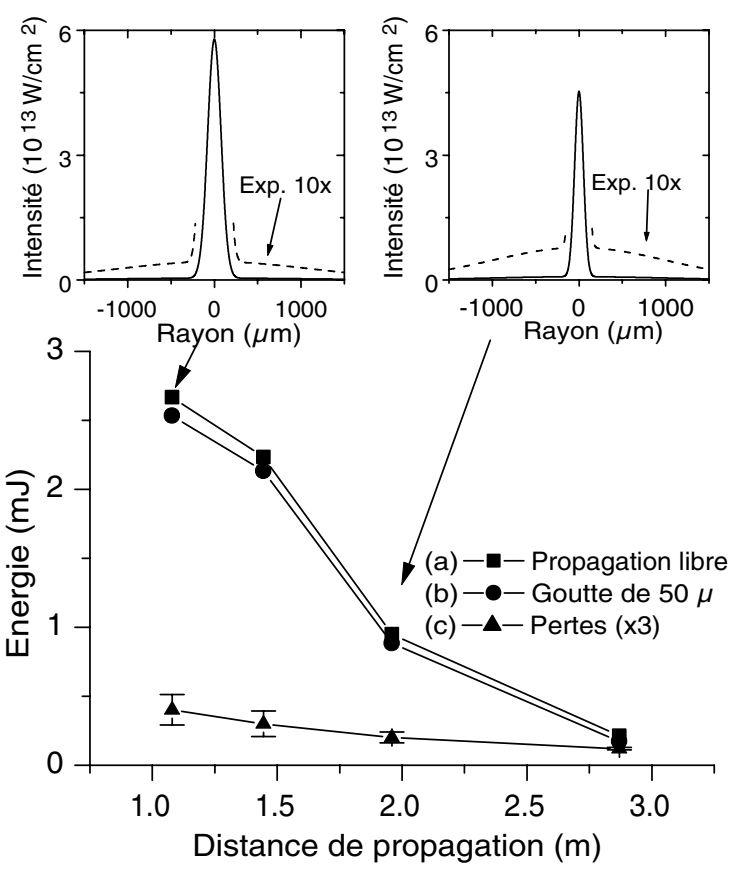

Figure 1. Interaction d'un filament autoguidé avec une goutte d'eau de $50 \mu \mathrm{m}$ de diamètre. (a) et (b): énergie contenue dans le filament en fonction de la distance de propagation $d$, comptée à partir du début du filament, (a) sans, et (b) en présence d'une goutte. Les inserts montrent le profil d'intensité du filament à deux distances, dans le cas d'une propagation libre. La courbe agrandie (x 10, en pointillés) montre la contribution du "bain de photons" entourant le filament. (c). énergie perdue par le filament à cause de l'interaction avec la goutte (soit la différence (a) - (b)): le filament regagne de l'énergie du bain au cours de sa propagation après l'interaction avec la goutte.

Après ces mesures préliminaires, nous avons placé une goutte de $50 \mu \mathrm{m}$ de diamètre au centre du filament, à une distance $d=1 \mathrm{~m}$ après le début de la filamentation. De manière étonnante, le filament n'est pas apparu perturbé par la présence de la goutte, bien que l'équilibre entre l'autofocalisation par effet Kerr et la défocalisation par le plasma joue un rôle critique à cause de la forte non-linéarité du processus de filamentation. De plus, la longueur du filament n'est pas affectée par l'interaction avec la goutte. Pour mieux comprendre ce résultat étonnant, nous avons mesuré l'énergie transportée dans le filament, en fonction de la distance de propagation afin de la comparer avec le cas de la propagation libre (Figure 1). La mesure à chaque distance est réalisée avec la même perforation du film pour les mesures avec et sans goutte. Ainsi, connaissant la précision de $\pm 1 \%$ sur les mesures d'énergie, on peut déterminer la précision sur la mesure de la perte d'énergie, $\Delta\left(E_{\text {libre }}-E_{\text {gouttes }}\right) /\left(E_{\text {libre }}-E_{\text {gouttes }}\right)$. On obtient entre $\pm 30 \%$ et $\pm 7 \%$, selon la valeur relative des pertes, comme le montrent les barres d'erreur portées sur la Figure 1 . Juste après l'interaction avec la goutte $(d=1 \mathrm{~m})$, la perte est limitée à $130 \pm 40 \mu \mathrm{J}$, mais l'équilibre est immédiatement rétabli. Par la suite, le filament semble regagner de l'énergie, la différence avec le filament non perturbé tombant à $40 \pm 3 \mu \mathrm{J}$ à la fin du filament $(d=3 \mathrm{~m})$. Ces mesures suggèrent que le filament reçoit du bain l'énergie nécessaire pour poursuivre sa propagation. Des prédictions théoriques [23,24], ainsi que de récentes simulations reproduisant les résultats expérimentaux [25,26], confirment cette interprétation en termes d'échanges d'énergie entre le filament et le "bain de photons" qui l'entoure.

Pour tester la robustesse de cet équilibre, nous avons reproduit l'expérience avec des gouttes plus grosses et opaques. Même des gouttes de $95 \mu \mathrm{m}$ de diamètre, qui bloquent l'essentiel de l'énergie contenue dans le filament, ne bloquent pas sa propagation, ni ne réduisent sa longueur (Figure 2). Les résultats sont similaires pour des gouttes transparentes (eau) et opaques (encre noire). La mesure des pertes d'énergie induites par les gouttes en fonction de $d$ confirme que le filament regagne de l'énergie $(0,2 \mathrm{~mJ}) \mathrm{du}$ 


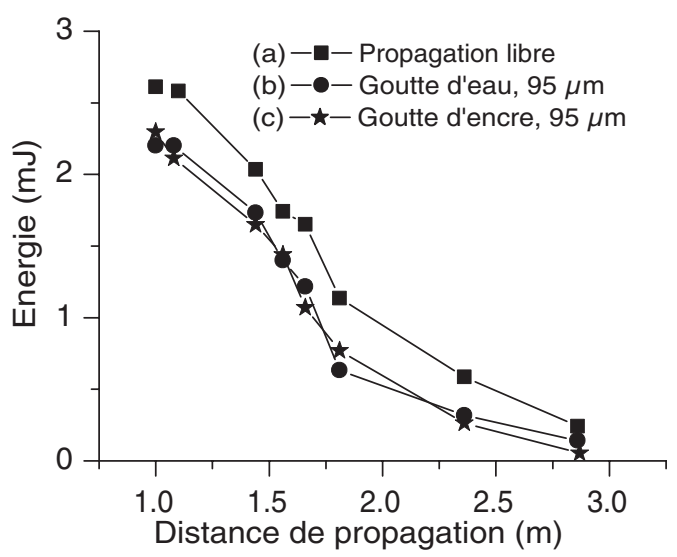

Figure 2. Rôle de la taille et de l'opacité de la goutte. Le filament survit à l'interaction avec une goutte de $95 \mu \mathrm{m}$ de diamètre, qu'il s'agisse d'eau (b), ou d'une goutte opaque (c). Les pertes d'énergie par rapport à la propagation libre (soit (c)-(a) et (b)-(a)) sont proportionnelles à la section efficace géométrique de la goutte.

"bain de photons" au cours de sa propagation. La perte immédiatement après l'interaction avec la goutte $(d=1 \mathrm{~m})$ est respectivement de $300 \pm 35$ et $370 \pm 35 \mu \mathrm{J}$ pour une goutte transparente et opaque de $95 \mu \mathrm{m}$. Ainsi, les pertes sont approximativement proportionnelles à la section efficace géométrique de l'obstacle inséré dans le filament. L'énergie de la zone du filament qui rencontre la goutte est diffractée sur l'obstacle, les interactions en volume dans la goutte ne jouant pas de rôle significatif. Ceci est confirmé par le fait que les pertes sont pratiquement insensibles à la composition de la goutte.

Ces expériences d'interaction du filament avec une goutte isolée démontrent que le filament est en équilibre avec le reste du faisceau. Le "bain de photons" entourant le filament lui fournit l'énergie nécessaire pour poursuivre sa propagation, voire le reconstitue tant que l'énergie restant dans le "bain" est suffisante. Ce résultat confirme l'observation selon laquelle un filament ne survit pas à la traversée d'un diaphragme qui bloque le "bain" [27].

\section{INTERACTION D'UN FILAMENT AVEC UN NUAGE}

La survie des filaments sur une longue distance à travers un nuage ou un brouillard sera donc conditionnée par l'extinction de l'énergie du "bain" par la diffusion élastique sur les gouttes d'eau du nuage. Pour caractériser cet effet, nous avons réalisé une seconde série d'expérience dans laquelle le filament se propageait dans une chambre à nuages longue de $35 \mathrm{~cm}$, ouverte aux deux extrémités pour laisser passer le faisceau laser. La distribution de taille des gouttes (diamètre moyen $4 \mu \mathrm{m}$, largeur à mi-hauteur de la distribution $2 \mu \mathrm{m}$ ) était mesurée grâce à la diffusion de Mie aux petits angles d'un laser Hélium-Néon. Simultanément, la transmission de ce même laser donnait accès à l'épaisseur optique $\tau=\ln (1 / T)$, où $T$ est la transmission. Simultanément, nous avons caractérisé la transmission du filament à travers le nuage et sa capacité à poursuivre sa propagation au-delà de la sortie du nuage.

La Figure 3 montre l'énergie du filament transmise à travers le nuage en fonction de $\tau$. Le filament se propage à travers le nuage jusqu'à une épaisseur optique de 3,2 (soit une concentration de gouttes de $410^{5} \mathrm{~cm}^{-3}$ ). Cependant, sa propagation au-delà du nuage ne dépasse alors pas quelques centimètres, à cause de la trop grande perte d'énergie du bain par diffusion élastique. À la sortie du nuage, son énergie ne suffit plus pour continuer à nourrir le filament. En effet, la puissance transmise, soit 2,3 GW, est inférieure à la puissance critique $P_{c}$ nécessaire pour que l'effet Kerr compense la diffraction. En revanche, pour une épaisseur optique de $1,2\left(10^{5}\right.$ gouttes $\left./ \mathrm{cm}^{3}\right)$, le filament transmis se propage normalement au-delà de la sortie du nuage, avec une longueur proche de celle observée en propagation libre. 


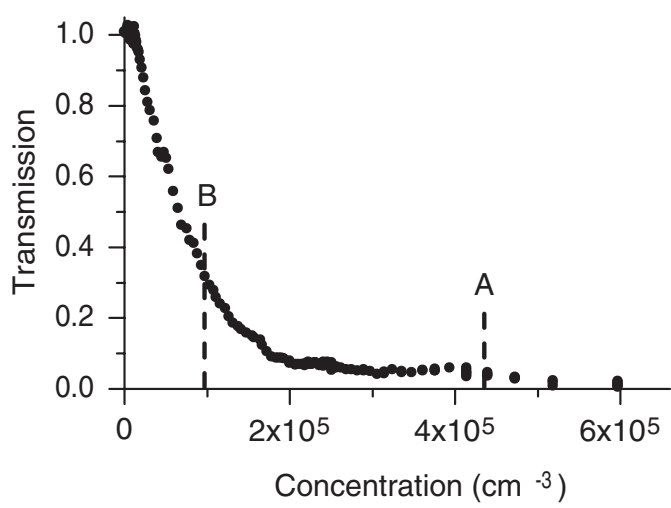

Figure 3. Transmission de filaments à travers un nuage de gouttes d'eau de diamètre moyen $4 \mu \mathrm{m}$. Le filament n'est pas significativement perturbé par le nuage jusqu'à une épaisseur optique $\tau=1,2\left(10^{5}\right.$ particules $/ \mathrm{cm}^{3}$, repère $\left.\mathbf{B}\right)$, et traverse le nuage, sans toutefois pouvoir se propager au-delà, jusqu'à $\tau=3,2$ (soit $410^{5}$ particules $/ \mathrm{cm}^{3}$, repère A).

Comme le montre la Figure 3, la transmission du filament décrô̂t exponentiellement avec la concentration de gouttes dans le nuage, comme on l'attend pour l'extinction par la diffusion élastique. Ainsi, les pertes d'énergie dans le bain par diffusion Mie dominent le processus et constituent la principale limite pour la transmission de filaments à travers des nuages et des brouillards. Cependant, l'épaisseur optique mesurée dans nos expériences est typique de nuages tels que des cumulus ou des stratocumulus [28].

\section{CONCLUSION}

Ces résultats sont donc particulièrement prometteurs pour les applications à l'atmosphère réelle, d'autant que les expériences ont été réalisées avec une énergie de $7 \mathrm{~mJ}$ par impulsion laser seulement. L'utilisation de laser de plus forte énergie tels que le Téramobile [29], premier laser femtoseconde-terawatt (400 mJ, $80 \mathrm{fs}$ ) mobile, pourrait conduire à des transmissions des filaments sur des épaisseurs optiques plus élevées encore. D'ores et déjà, des expériences en laboratoire ont montré que le déclenchement et le guidage par laser de décharges de haute tension restent possible sur 1,2 m, sous un flux de gouttes d'eau correspondant à une hauteur de précipitation de $1,4 \mathrm{~mm} / \mathrm{min}$, correspondant à une forte pluie [30]. Il s'agit à ce jour des simulations en laboratoire les plus réalistes pour le déclenchement de foudre par laser.

\section{Remerciements}

Les auteurs remercient le CNRS, le Ministère de la Recherche, l'Institut Universitaire de France et le projet francoallemand Téramobile, co-financé par le CNRS et la DFG, pour leur soutien financier. Nous remercions vivement M. Néri et M. Kerleroux pour le support technique.

\section{Bibliographie}

[1] D. W. Koopman, T. D. Wilkerson, J. Appl. Phys. 42, 1883 (1971)

[2] M. Miki, Y. Aihara, T. Shindo, J. Phys. D: Appl. Phys. 26, 1244-1252 (1993)

[3] X. M. Zhao, J.-C. Diels, C. Y. Wang, J. M. Elizondo, IEEE J. Quantum Electron. 31, 599 (1995)

[4] H. Pépin, D. Comptois, F. Vidal, C. Y. Chien, A. Desparois, T. W. Johnston, J. C. Kieffer, B. La Fontaine, F. Martin, F. A. M. Rizk, C. Potvin, P. Couture, H. P. Mercure, A. BondiouClergerie, P. Lalande et I. Gallimberti, Phys. Plasmas 8, 2532 (2001) 
[5] D. Comptois, C. Y. Chien, A. Desparois, F. Gérin, G. Jarry, T. W. Johnston, J. C. Kieffer, B. La Fontaine, F. Martin, R. Mawassi, H. Pépin, F. A. M. Rizk, F. Vidal, P. Couture, H. P. Mercure, C. Potvin, A. Bondiou-Clergerie, I. Gallimberti, Appl. Phys. Lett. 76, 819 (2000)

[6] D. Comtois, H. Pépin, F. Vidal, F. A. M. Risk, C.-Y. Chien, T. W. Johnston, J.-C. Kieffer, B. La Fontaine, F. Martin, C. Potvin, P. Couture, H. P. Mercure, A. Bondiou-Clergerie, P. Lalande, I. Gallimberti, IEEE Trans. Plasma Sci. 31, 377 (2003)

[7] D. Comtois, H. Pépin, F. Vidal, F. A. M. Risk, C.-Y. Chien, T. W. Johnston, J.-C. Kieffer, B. La Fontaine, F. Martin, C. Potvin, P. Couture, H. P. Mercure, A. Bondiou-Clergerie, P. Lalande, I. Gallimberti, IEEE Trans. Plasma Sci. 31, 387 (2003)

[8] B. La Fontaine, D. Comptois, C. Y. Chien, A. Desparois, F. Gérin, G. Jarry, T. W. Johnston, J. C. Kieffer, F. Martin, R. Mawassi, H. Pépin, F. A. M. Rizk, F. Vidal, C. Potvin, P. Couture, H. P. Mercure, J. Appl. Phys. 88, 610 (2000)

[9] M. Rodriguez, R. Sauerbrey, H. Wille, L. Wöste, T. Fujii, Y.-B. André, A. Mysyrowicz, L. Klingbeil, K. Rethmeier, W. Kalkner, J. Kasparian, E. Salmon, J. Yu, J.-P. Wolf, Opt. Lett. 27, 772 (2002)

[10] B. La Fontaine, F. Vidal, Z. Jiang, C. Y. Chien, D. Comtois, A. Desparois, T. W. Johnson, J.-C. Kieffer et H. Pépin, Phys. Plasmas 6, 1615 (1999).

[11] M. Rodriguez, R. Bourayou, G. Méjean, J. Kasparian, J. Yu, E. Salmon, A. Scholz, B. Stecklum, J. Eislöffel, U. Laux, A. P. Hatzes, R. Sauerbrey, L. Wöste et J.-P. Wolf, Phys. Rev. E 69, 036607 (2004)

[12] S. Tzortzakis, B. Prade, M. Franco et A. Mysyrowicz, Opt. Commun. 181, 123 (2000)

[13] P. Rairoux, H. Schillinger, S. Niedermeier, M. Rodriguez, F. Ronneberger, R. Sauerbrey, B. Stein, D. Waite, C. Wedekind, H. Wille, L. Woste, C. Ziener, Appl. Phys. B 71, 573 (2000)

[14] J. Kasparian, M. Rodriguez, G. Méjean, J. Yu, E. Salmon, H. Wille, R. Bourayou, S. Frey, Y.-B. André, A. Mysyrowicz, R. Sauerbrey, J.-P. Wolf et L. Wöste, Science 301, 61 (2003)

[15] L. Iorio, Classical and Quantum Gravity 19, 175 (2002)

[16] G. A. Askar'yan, Sov. Phys. JETP 15, 1088 (1962)

[17] R. Y. Chiao, E. Garmire et C. H. Townes, Phys. Rev. Lett. 13, 479 (1964)

[18] A. Braun, G. Korn, X. Liu, D. Du, J. Squier, G. Mourou, Opt. Lett. 20, 73 (1995).

[19] L. Bergé, A. Couairon, Phys. Rev. Lett. 86, 1003 (2001)

[20] W. Liu, S. Petit, A. Becker, N. Aközbek, C. M. Bowden et S. L. Chin, Opt. Commun. 202, 189 (2002)

[21] F. Courvoisier, V. Boutou, J. Kasparian, E. Salmon, G. Méjean, J. Yu, et J. P. Wolf, Appl. Phys. Lett. 83, 213 (2003)

[22] C. Favre, V. Boutou, S. C. Hill, W. Zimmer, M. Krenz, H. Lambrecht, J. Yu, R. K. Chang, L. Woeste, J. P. Wolf, Phys. Rev. Lett. 89035002 (2002)

[23] M. Mlejnek, E. M. Wright, J. V. Moloney, Opt. Lett. 23, 382 (1998)

[24] M. Mlejnek, E. M. Wright, J. V. Moloney, Opt. Express 4, 223 (1999)

[25] M. Kolesik, J. V. Moloney, Opt. Lett. 29, 590 (2004)

[26] S. Skupin, L. Bergé, U. Peschel et F. Lederer, Phys. Rev. Lett. 93, 023901 (2004)

[27] S. L. Chin, A. Brodeur, S. Petit, O. G. Kosareva, V. P. Kandidov, J. Nonlinear Opt. Phys. Mater. 8, 121 (1999)

[28] Classification du World Climate Research Program (WCRP). Voir par exemple : http://isccp.giss. nasa.gov/cloudtypes.html

[29] H. Wille, M. Rodriguez, J. Kasparian, D. Mondelain, J. Yu, A . Mysyrowicz, R. Sauerbrey, J. P. Wolf, L. Wöste, Eur. Phys. J.- Appl. Phys. 20, 183 (2002)

[30] R. Ackermann, K. Stelmasczyck, P. Rohwetter, G. Méjean, E. Salmon, J. Yu, J. Kasparian, G. Méchain, K. Rethmeier, W. Kalkner, J. P. Wolf, L. Wöste, Appl. Phys. Lett. 85, 5781 (2004) 